\title{
Control of an Pseudo-omnidirectional, Non-holonomic, Mobile Robot based on an ICM Representation in Spherical Coordinates
}

\author{
Christian P. Connette, Andreas Pott, Martin Hägele, Alexander Verl
}

\begin{abstract}
For mobile platforms with steerable standard wheels it is necessary to precisely coordinate rotation and steering angle of their wheels. Especially for redundantly actuated platforms the misalignments of a single wheel directly leads to invalid configurations which may cause degraded motion of the platform and high internal forces. An established approach to deal with this problem is to represent the current state of motion in form of the Instantaneous Centre of Motion (ICM) and to derive a valid trajectory for this point. However, this representation bears severe numerical drawbacks.

To remedy those numerical problems an alternative ICM representation based on spherical coordinates is proposed in this work. Furthermore, the relations between ICM and generalized robot velocities are addressed. It is shown, that one receives a basis of a subspace within the kinematical constraints' nullspace by decomposing the generalized velocity vector in spherical coordinates. Finally the proposed ICM-based control is particularized and simulative analyzed w.r.t. the CareO-bot 3 demonstrator (Fig. 1).
\end{abstract}

\section{INTRODUCTION}

An essential prerequisite for future service robots is a safe and efficient operation amongst humans [1]. Thus a high degree of mobility, flexibility and robustness of the mobile platforms is required. Accordingly a wide variety of different motion concepts exists ranging from snake-like approaches over wheeled platforms up to humanoid robot systems [2], [3]. A very intelligible introduction can be found in [4]. Currently wheeled platforms appear to be a promising compromise between a high degree of flexibility and robustness on the one hand and moderate complexity on the other hand. Therefore many efforts were taken to design, build and control a diversity of different wheeled systems [5], [6]. The approaches range from simple differential driven platforms and systems with centered or offcentered orientable wheels to mobile robots that use specially designed wheels [7], [8], like the Swedish or the orb wheel. Orientable wheels offer high robustness and comparably small mechanical complexity on the one hand, on the other the systems flexibility is slightly reduced and the complexity of the control scheme is increased.

In their seminal work [5] Campion et al. categorized all wheeled mobile robots by their kinematic properties into five different classes. They expressed all kinematic constraints in a matrix $M$. The entirety of all allowed configurations of the mobile platform - all configurations that fulfill the kinematic

This work was conducted in the department for Robotic Systems at the Fraunhofer Institute for Manufacturing Engineering and Automation (IPA), 70504 Stuttgart, Germany - The work was partially conducted within the EU Project COGNIRON ("The Cognitive Robot Companion" - www.cogniron.org) under Contract FP6-IST-FET-002020.

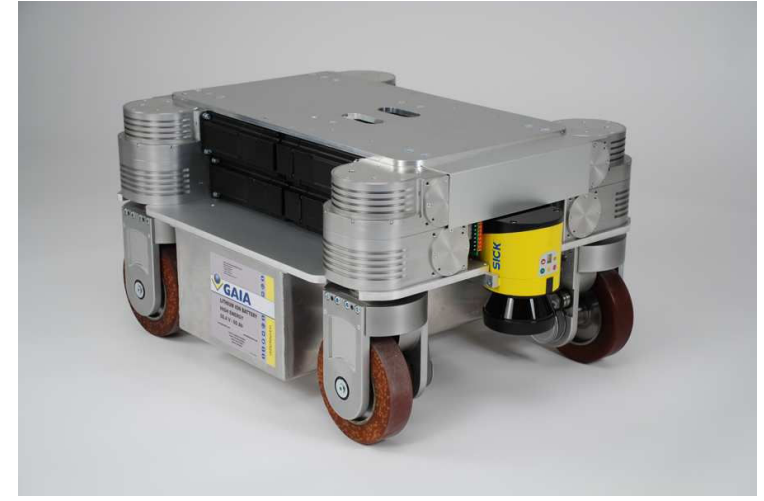

Fig. 1. Mobile Base of Care-O-bot 3 (www.care-o-bot.de)

constraints - is then formed by the nullspace $N[M]$ of $M$. The dimension of the nullspace " $\operatorname{dim} N[M]$ " and the rank of $M$ "rank $[M]$ " classify the kinematic properties of the system. The corresponding numbers are usually referred to as the degree of mobility

$$
\delta_{\mathrm{m}}=\operatorname{dim} N[M]=3-\operatorname{rank}[M]
$$

and the degree of steerability

$$
\delta_{\mathrm{S}}=\operatorname{rank}[M] .
$$

$\delta_{\mathrm{m}}$ resembles the dimension of the instantaneously accessible velocity space - also called the differentiable degrees of freedom [4] - while $\delta_{\mathrm{S}}$ corresponds to the number of independently steerable wheels. For any mobile platform with two or more orientable wheels $\delta_{\mathrm{m}}$ equals 1 and $\delta_{\mathrm{S}}$ equals 2 for any non-degenerated configuration, i.e. any configuration that allows the robot to move. This implies that a robot with orientable wheels cannot instantaneously change its driving direction and that all wheels have to be precisely coordinated.

A basic strategy - given slowly changing set point velocities and a sufficiently high control frequency - is to calculate every wheel's steering direction and velocity by superposition of the set point velocities [9]. However, this strategy encounters some problems, as the set point values for the steering directions depend on the ratios of the velocity components. Thus, especially if the velocity components are small, already minor changes of these components have a high impact on the related set point values. A strategy providing an accurate solution to the problem is to calculate an optimal path within the nullspace of the kinematic constraints. However, this can become a very complex task, 
especially if the nonlinear character and singularities of the system are considered.

Besides a wide variety of different, other approaches [10], [11], an often applied middle course between above mentioned methods is to calculate the Instantaneous Centre of Motion (ICM) [12], [13], [14] and derive a valid trajectory for it. However, this ICM-based representation has some numerical drawbacks, which affect potential control strategies. A detailed discussion of these problems can be found in [15], [16].

This work approaches the problem of wheel coordination through control of the ICM. It gives a short introduction to the ICM (section II) and addresses the numerical drawbacks. Similar to [15] an alternative parameterization of the ICM, which remedies these problems, is proposed. The basic idea of this approach is to relocate critical singularities in non-critical regions of the ICM's parameter-space. It is shown that, in a conveniently defined coordinate system, the proposed representation is closely related to a spherical representation of the system's generalized velocities, respectively the twist vector

$$
\vec{t}=\left(\begin{array}{c}
v_{x} \\
v_{y} \\
\omega
\end{array}\right)
$$

Furthermore, the transformation equations from the defined ICM space into the robots configuration space are derived. Finally, the proposed control scheme is particularized and analyzed with respect to the control architecture of Care-Obot 3 .

\section{ICM DEFINITION AND NUMERICAL ISSUES}

\section{A. Definition}

There are several corresponding definitions for the Instantaneous Centre of Motion (ICM). Within this work the ICM is defined as the point in the world coordinate frame, which instantaneously does not change with respect to the robot, while the latter is moving. It is the point around which the robot rotates, the centre of the generalized curve on which the robot moves at the very moment. For mobile robots with steered standard wheels it is furthermore the point where all wheel axis intersect. However, the last statement only holds if the kinematic constraints are not violated e.g. no slipping occurs. In the world coordinate frame the ICM position can be calculated as

$$
\vec{x}_{\mathrm{ICM}}^{w}=\left(\begin{array}{c}
x_{\mathrm{ICM}}^{w} \\
y_{\mathrm{ICM}}^{w}
\end{array}\right)=\left(\begin{array}{c}
x_{r}^{w}-v_{r, y}^{w} / \omega_{r}^{w} \\
y_{r}^{w}+v_{r, x}^{w} / \omega_{r}^{w}
\end{array}\right),
$$

where $x_{r}^{w}, y_{r}^{w}$ and $\theta_{r}^{w}$ are components of the robots position and orientation vector $\vec{x}_{r}^{w}$ and $v_{r, x}^{w}, v_{r, y}^{w}, \omega_{r}^{w}$ are the components of the robots twist $\vec{t}_{r}^{w}$

$$
\vec{x}_{r}^{w}=\left(\begin{array}{c}
x_{r}^{w} \\
y_{r}^{w} \\
\theta_{r}^{w}
\end{array}\right), \vec{t}_{r}^{w}=\left(\begin{array}{c}
v_{r, x}^{w} \\
v_{r, y}^{w} \\
\omega_{r}^{w}
\end{array}\right)
$$

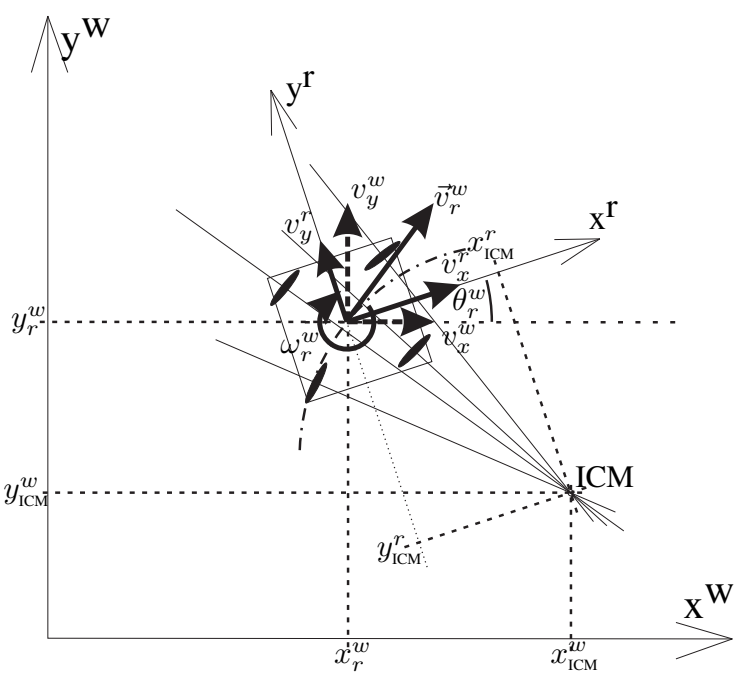

Fig. 2. Applied Coordinate Systems and ICM

in the world frame (Fig. 2). Transformation to the robot coordinate frame delivers

$$
\vec{x}_{\mathrm{ICM}}^{r}=\left(\begin{array}{c}
x_{\mathrm{ICM}}^{r} \\
y_{\mathrm{ICM}}^{r}
\end{array}\right)=\left(\begin{array}{c}
-v_{r, y}^{r} / \omega_{r}^{r} \\
+v_{r, x}^{r} / \omega_{r}^{r}
\end{array}\right)
$$

for the ICM position, where $v_{r, x}^{r}, v_{r, y}^{r}$ and $\omega_{r}^{r}$ are the components of the robot's twist $\overrightarrow{t_{r}^{r}}$ w.r.t the world frame

$$
\vec{t}_{r}^{r}=\left(\begin{array}{c}
v_{r, x}^{r} \\
v_{r, y}^{r} \\
\omega_{r}^{r}
\end{array}\right)=\left(\begin{array}{c}
v_{r, x}^{w} \cos \left(\theta_{r}^{w}\right)+v_{r, y}^{w} \sin \left(\theta_{r}^{w}\right) \\
-v_{r, x}^{w} \sin \left(\theta_{r}^{w}\right)+v_{r, y}^{w} \cos \left(\theta_{r}^{w}\right) \\
\omega_{r}^{w}
\end{array}\right)
$$

expressed in the robot coordinate frame. As we are not interested in the global behavior of the robot we confine our reflections on the robot-coordinate frame. Following all velocities and positions are expressed relative to the robotcoordinate frame. For a more convenient writing we thus omit the indicators of the coordinate system and write $\vec{t}_{r}$ instead of $\vec{t}_{r}^{r}$ and $\vec{x}_{\mathrm{ICM}}$ instead of $\vec{x}_{\mathrm{ICM}}^{r}$ respectively.

\section{B. Numerical Issues}

The numerical problems arising while using an ICMbased representation of the twist $\vec{t}$ are twofold. The first problem is that the transformation $f_{\mathrm{ICM}}\left(\vec{t}_{r}\right)$ from the vector representation of the twist $\vec{t}_{r}$ into the ICM representation (1),(2) is not injective. Thus, information is lost and the ICM cannot be transformed back directly. The second, more severe problem is the singularity arising when $\omega_{r}$ becomes zero (Fig. 3).The right-side limit then becomes

$$
\lim _{\omega_{r} \rightarrow 0^{+}} f_{\mathrm{ICM}}\left(\vec{t}_{r}\right) \rightarrow+\infty
$$

while the left-side limit is

$$
\lim _{\omega_{r} \rightarrow 0^{-}} f_{\text {ICM }}\left(\vec{t}_{r}\right) \rightarrow-\infty
$$

and thus are not equal (Fig. 4(a)). This singularity however, is introduced only by the chosen parameterization and does not have any physical meaning. It occurs in a frequently traversed region of the parameter space and thus hinders controller design. 


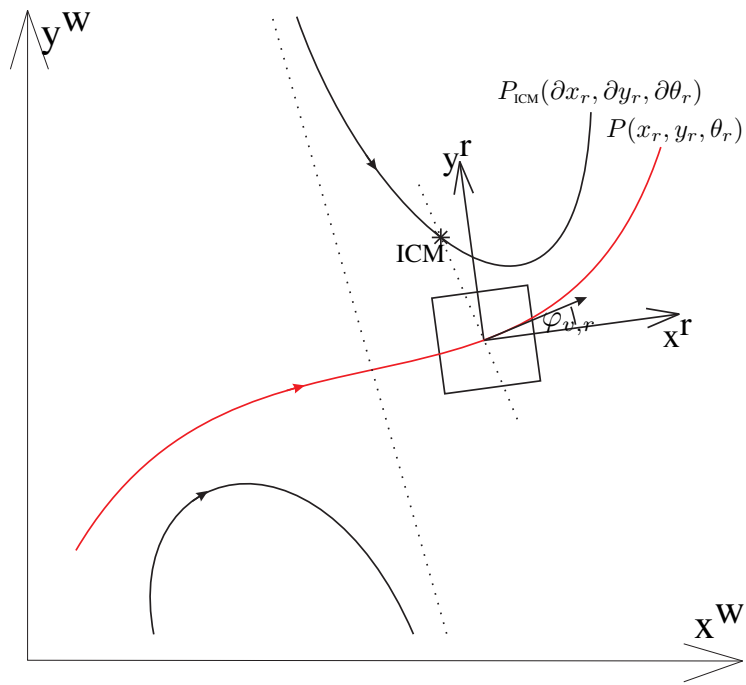

Fig. 3. Example for an arbitrary trajectory of robot and ICM

\section{ICM IN SPHERICAL COORDINATES}

The following representation adapts the formulation of the ICM addressing singularity by relocation and consering all available information. Before introducing the new parameterization for the ICM a coordinate system with the basis $v_{x}, v_{y}$ and $\omega \cdot d_{\max }$ is defined to represent the robot's motion. The constant factor $d_{\max }$ is introduced to render the rotational velocity the same dimension as the translational velocities. Accordingly the robot twist $\vec{t}_{r}$ is redefined to

$$
\overrightarrow{t_{r}^{*}}=\left(\begin{array}{c}
v_{r, x} \\
v_{r, y} \\
\omega_{r} \cdot d_{\max }
\end{array}\right)
$$

\section{A. Conserving all available Information}

Transforming the Cartesian representation (2) of the ICM into polar coordinates

$$
\left(\begin{array}{c}
r_{\text {ICM }} \\
\phi_{\text {ICM }}
\end{array}\right)=\left(\begin{array}{c}
\frac{\sqrt{v_{r, x}^{2}+v_{r, y}^{2}}}{\left|\omega_{r}\right|} \\
\arctan _{2}\left(\frac{v_{r, x} / \omega_{r}}{-v_{r, y} / \omega_{r}}\right)
\end{array}\right),
$$

replacing $\left|\omega_{r}\right|$ with $\omega_{r}$, removing $\omega_{r}$ from the $\phi_{\text {ICM }}$ term

$$
\left(\begin{array}{c}
r_{\text {ICM }} \\
\phi_{\text {ICM }}
\end{array}\right):=\left(\begin{array}{c}
\frac{\sqrt{v_{r, x}^{2}+v_{r, y}^{2}}}{\omega_{r}} \\
\arctan _{2}\left(\frac{v_{r, x}}{-v_{r, y}}\right)
\end{array}\right)
$$

and accepting the peculiarity that the distance $r_{\text {Iсм }}$ may take negative values, allows to regain the signs of all velocity components. To recover also the absolute values of the components of the twist $\vec{t}^{*}$ the absolute translational velocity $v_{\text {abs }}=\left(v_{r, x}^{2}+v_{r, y}^{2}\right)^{-\frac{1}{2}}$ can be used. However, to achieve a convenient formulation with respect to the above defined three-dimensional coordinate system the term $\omega_{r} \cdot d_{\max }$ is added:

$$
v_{\mathrm{abs}}^{*}:=\sqrt{v_{r, x}^{2}+v_{r, y}^{2}+\left(\omega_{r} \cdot d_{\max }\right)^{2}}
$$

\section{B. Relocating the Singularities}

The above derived equations do not tackle the problem of the infinity points of the ICM. To tackle that problem a new parameter

$$
\theta_{\mathrm{ICM}}:=\arctan \left(\frac{d_{\max }}{r_{\mathrm{ICM}}}\right)
$$

is defined. Calculating the inverse to $r_{\text {ICM }}$ corresponds to a reflection over the unit circle. This relocates $\pm \infty$-regions (Fig. 4(a)) to the origin. The region around the origin (Fig. 4) is vice versa relocated at infinity. The calculation of the arctan resembles a projection of the infinite plane on a spherical surface. Thus, the mentioned singularities are eliminated. Only if the absolute velocity is close to zero small fluctuations in $\omega_{r}$ will cause significant jumps (Fig. 4(c)). However, in contrast to the former representation the parameters now stay bounded.

\section{Correlation to and Relevancy for the Velocity-Space}

By defining the new parameter $\varphi_{\text {ICM }}$ by adding $\frac{\pi}{2}$ to the $\phi_{\mathrm{ICM}}$-term in Equation (4) and by renaming $v_{t o t}^{*}$ in (5) as $\rho_{\mathrm{ICM}}$ we get:

$$
\begin{aligned}
\rho_{\text {ICM }} & :=\sqrt{v_{r, x}^{2}+v_{r, y}^{2}+\left(\omega_{r} \cdot d_{\text {max }}\right)^{2}} \\
\varphi_{\text {ICM }} & :=\arctan _{2}\left(\frac{v_{r, y}}{v_{r, x}}\right) \\
\theta_{\text {ICM }} & :=\arctan \left(\frac{\omega_{r} \cdot d_{\text {max }}}{\sqrt{v_{r, x}^{2}+v_{r, y}^{2}}}\right)
\end{aligned}
$$

as the resulting parameterization of the ICM. This formulation is identical to the representation of the twist vector $\vec{t} *$ in spherical coordinates. Thus, the backwards transformation

$$
\begin{aligned}
v_{r, x} & =\rho_{\mathrm{ICM}} \cdot \cos \left(\theta_{\mathrm{ICM}}\right) \cdot \cos \left(\varphi_{\mathrm{ICM}}\right) \\
v_{r, y} & =\rho_{\mathrm{ICM}} \cdot \cos \left(\theta_{\mathrm{ICM}}\right) \cdot \sin \left(\varphi_{\mathrm{ICM}}\right) \\
\omega_{r} \cdot d_{\text {max }} & =\rho_{\mathrm{ICM}} \cdot \sin \left(\theta_{\mathrm{ICM}}\right)
\end{aligned}
$$

is directly given by the standard transformation between spherical and Cartesian coordinates. As the transformations applied on (4) conserve neighborhood relations, any continuous trajectory in the spherical representation transforms into a continuous trajectory in the originating, planar ICM space. This means, that the above defined three dimensional ICM space also represents a subspace of the nullspace $N[M]$ of the kinematic constraints. As the parameterization of the above defined ICM is identical with the representation of the twist vector $\vec{t}^{*}$ in spherical coordinates, any continuous change in the spherical coordinates of $\overrightarrow{t^{*}}$ represents a valid trajectory within the nullspace of the robot's kinematical constraints. Descriptively this implies that a valid trajectory within $N[M]$ can always be found by independently controling the spherical coordinates of the twist $\vec{t}^{*}$. It has to be noted however, that this representation does not account for other system immanent singularities or other boundary conditions, e.g. restrictions on the steering rates. Thus, a valid control strategy has to consider these additional implications. 


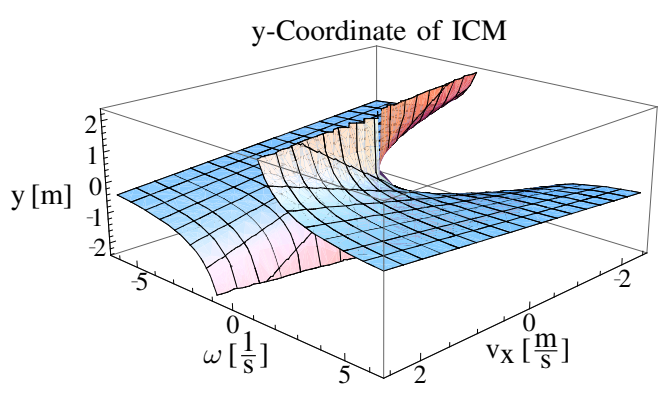

(a) Region around Singularity for $\lim _{\omega_{r} \rightarrow 0} f_{\mathrm{ICM}}\left(\vec{t}_{r}\right)$

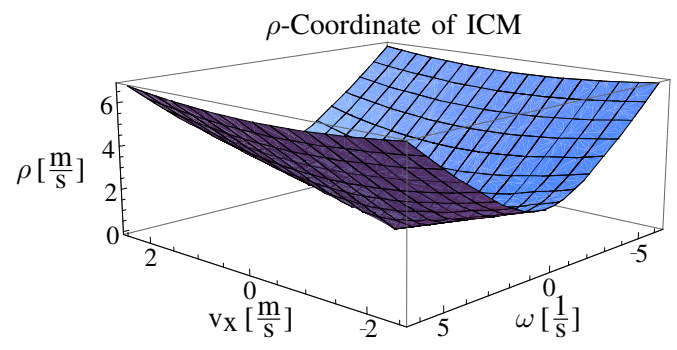

(b) No singularities for $\rho$ in same region

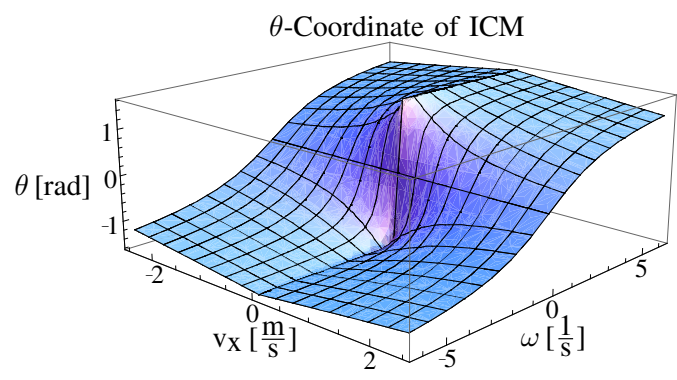

(c) Only singularity of $\theta$ for $v \equiv 0$ and $\omega \equiv 0$

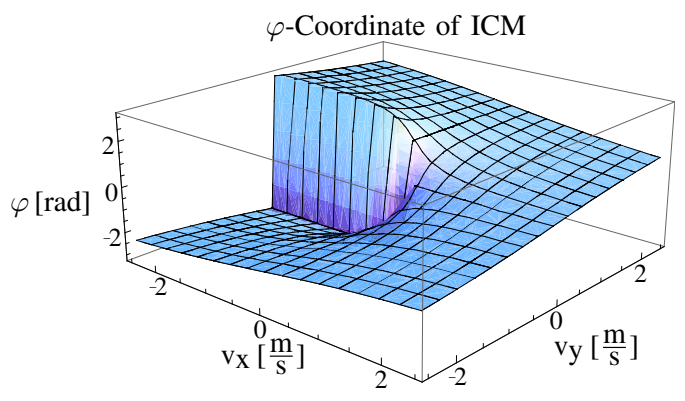

(d) Unsteadiness of Transformation to $\varphi$

Fig. 4. Visualization of Singularities in same region for the different Transformations from Velocity to ICM space

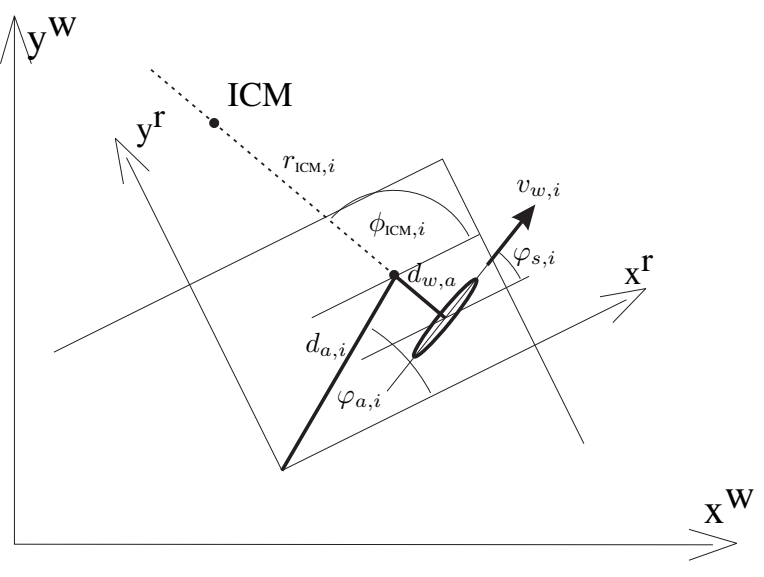

Fig. 5. Wheel-Axis based parameterization

\section{Retaining the Wheel Set Point Values}

This section delivers a definition for the transformation $f_{\vec{v}, \vec{\varphi}}\left(f_{\text {ICM }}\left(\vec{t}_{r}^{*}\right)\right)$ from ICM-configuration to the resulting platform configuration, namely velocities $\vec{v}$ and steering angles $\vec{\varphi}$ of the wheels. The backward transformation is written as $f_{\vec{v}, \vec{\varphi}}^{-1 *}$, although as indicated in section II a direct inversion is not possible. Based on all wheel velocities $\vec{v}_{w}$ and steering angles $\vec{\varphi}_{s}$ only an estimation of the current ICM can be calculated.

\section{A. Definition}

Let $\varphi_{s, i}$ be the steering direction and $v_{w, i}$ the velocity of the $i$ th wheel (Fig. 5). The position of the wheel's steering axis $\vec{x}_{a, i}$ and $\vec{x}_{a, i}^{*}$ in the robot coordinate-system is given as

$$
\vec{x}_{a, i}=\left(\begin{array}{c}
x_{a, i} \\
y_{a, i}
\end{array}\right), \vec{x}_{a, i}^{*}=\left(\begin{array}{c}
d_{a, i} \\
\varphi_{a, i}
\end{array}\right)
$$

in Cartesian coordinates and in polar coordinates respectively. The offset between wheel steering axis and wheel center is given by $d_{w a}$. The wheel's position $\left(x_{w, i}, y_{w, i}\right)$ itself is calculated via

$$
\begin{aligned}
x_{w, i} & =x_{a, i}+d_{w, a} \sin \left(\varphi_{s, i}\right) \\
y_{w, i} & =y_{a, i}-d_{w, a} \cos \left(\varphi_{s, i}\right)
\end{aligned}
$$

The planar ICM position, relative to the wheel's steering axis, is given by the angle $\phi_{\mathrm{ICM}, i}$ and $r_{\mathrm{Icm}, i}$. It is furthermore defined a spherical ICM representation $\left(\rho_{\mathrm{ICM}, w_{i}}, \varphi_{\mathrm{ICM}, w_{i}}, \theta_{\mathrm{ICM}, w_{i}}\right)$ relative to the current wheel position, which transforms into the generalized wheel velocities $\left(v_{x, w_{i}}, v_{y, w_{i}}, \omega_{w_{i}} d_{\max }\right)$. The according transformation $\vec{f}_{\mathrm{ICM}, w}\left(v_{x, w_{i}}, v_{y, w_{i}}, \omega_{w_{i}}\right)$ is defined by the equations (7) to (12).

\section{B. Transforming ICM to Wheel Configuration}

To obtain the wheel related ICM the rigid body constraint for the robot is exploited to express the wheel velocities as a function of the robot velocities:

$$
\begin{aligned}
v_{x, w_{i}} & =v_{x, r}-y_{w, i} \omega_{r} \\
v_{y, w_{i}} & =v_{y, r}+x_{w, i} \omega_{r} \\
\omega_{w_{i}} & =\omega_{r}
\end{aligned}
$$


Inserting the dependencies (15) - (17) into equations (7) (9) for the wheel related ICM and subsequent simplification delivers the transformation $\vec{g}_{\mathrm{ICM}, w_{i}}\left(\rho_{\mathrm{ICM}}, \varphi_{\mathrm{ICM}}, \theta_{\mathrm{ICM}}\right)$ from robot related ICM to wheel related ICM. Simplifying the derived equations by using that $\rho_{\mathrm{ICM}} \geq 0$ delivers

$$
\begin{aligned}
& \rho_{\mathrm{ICM}, w_{i}}=\rho_{\mathrm{ICM}} \cdot \sqrt{a^{2}+b^{2}+c^{2}} \\
& \varphi_{\mathrm{ICM}, w_{i}}=\arctan _{2}\left(\frac{b}{a}\right) \\
& \theta_{\mathrm{ICM}, w_{i}}=\arctan \left(\frac{c}{\sqrt{a^{2}+b^{2}}}\right)
\end{aligned}
$$

with the components $a, b$ and $c$, calculated via

$$
\begin{aligned}
a & =\cos \left(\theta_{\mathrm{ICM}}\right) \cos \left(\varphi_{\mathrm{ICM}}\right)-\frac{y_{w, i}}{d_{\max }} \sin \left(\theta_{\mathrm{ICM}}\right) \\
b & =\cos \left(\theta_{\mathrm{ICM}}\right) \sin \left(\varphi_{\mathrm{ICM}}\right)+\frac{x_{w, i}}{d_{\max }} \sin \left(\theta_{\mathrm{ICM}}\right) \\
c & =\sin \left(\theta_{\mathrm{ICM}}\right) .
\end{aligned}
$$

In the case of steerable standard wheels without sideward offset the wheel position $\left(y_{w, i}, x_{w, i}\right)$ can be substituted by the constant axis position $\left(y_{a, i}, x_{a, i}\right)$. As fulfilling the kinematical constraints implies that wheel, wheel steering axis and ICM must lie on one line this substitution is also valid when calculating the wheel steering directions $\varphi_{\mathrm{ICM}, w_{i}}$ according to equation (19). Applying the backwards transformation $\vec{f}_{\mathrm{ICM}, w}^{-1}\left(v_{x, w_{i}}, v_{y, w_{i}}, \omega_{w_{i}}\right)$ defined by $(10)-(12)$ on the calculated ICM parameters delivers the generalized velocities of the wheel. The corresponding wheel configuration is then calculated by

$$
\begin{aligned}
& \varphi_{s, i}=\arctan _{2}\left(\frac{v_{y, w_{i}}}{v_{x, w_{i}}}\right)+k_{1} \\
& v_{\text {lin }, i}=k_{2} \cdot \sqrt{v_{x, w_{i}}^{2}+v_{y, w_{i}}^{2}}
\end{aligned}
$$

where the two factors $k_{1}$ and $k_{2}$ are introduced to account for the ambiguity of the wheel configuration

$$
\left\{k_{1} ; k_{2}\right\}=\left\{\begin{array}{cc}
\{0 ;+1\} & \left|\varphi_{s, i, \text { desired }}-\varphi_{s, i, \text { meas }}\right| \leq \frac{\pi}{2} \\
\{\pi ;-1\} & \text { otherwise }
\end{array}\right.
$$

and the corresponding branching of the solutions. The wheel velocity $v_{w, i}$ is finally obtained by adding a systemdependent compensation term $v_{\text {comp }, i}$

$$
v_{w, i}=v_{\text {lin }, i}+v_{\text {comp }, i}
$$

to account for gear-coupling and the motion of the wheels around their steering axis.

Concatenating the derived transformations from robot ICM to wheel $\operatorname{ICM}(18,19,20)$, wheel ICM to generalized wheel velocities $(10,11,12)$ and generalized wheel velocities to wheel configuration $(21,22,23)$ for all wheels one obtains the transformation

$$
\left(\begin{array}{c}
\varphi_{s, 1} \\
v_{w, 1, \text { lin }} \\
\vdots \\
\varphi_{s, n} \\
v_{w, n, l \text { lin }}
\end{array}\right)=\vec{f}_{\vec{v}, \vec{\varphi}}\left(\varphi_{\text {IсM }}, \theta_{\mathrm{ICM}}, \rho_{\mathrm{ICM}}\right)
$$

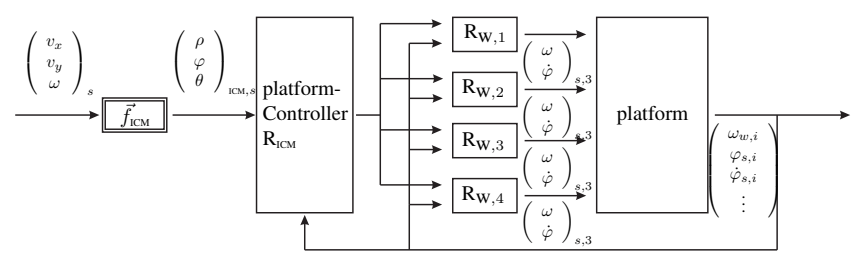

Fig. 6. Overall control structure of the platform

from the robot's spherical ICM to the platform configuration space. The corresponding derivatives in the configuration space are obtained by incorporating the according Jacobian $J_{\vec{v}, \vec{\varphi}}$

$$
\left(\begin{array}{c}
\dot{\varphi}_{w 1, s} \\
\dot{v}_{w 1, l i n} \\
\vdots \\
\dot{\varphi}_{w n, s} \\
\dot{v}_{w n, l i n}
\end{array}\right)=\underbrace{\frac{\vec{f}_{\vec{v}, \vec{\varphi}}}{\partial\left(\varphi_{\mathrm{ICM}}, \theta_{\mathrm{ICM}}, \rho_{\mathrm{ICM}}\right)}}_{J_{\vec{v}, \vec{\varphi}}\left(\varphi_{\mathrm{ICM}}, \theta_{\mathrm{ICM}}, \rho_{\mathrm{ICM}}\right)} \cdot\left(\begin{array}{c}
\dot{\varphi}_{\mathrm{ICM}} \\
\dot{\theta}_{\mathrm{ICM}} \\
\dot{\rho}_{\mathrm{ICM}}
\end{array}\right) .
$$

Analogous the Jacobian $J_{\vec{v}, \vec{\varphi}}^{-1 *}$ for the approximate backward transformation $\vec{f}_{\vec{v}, \vec{\varphi}}^{-1 *}$ and the Jacobian $J_{\text {ICM }}$ for the transformation $\vec{f}_{\mathrm{ICM}}\left(v_{r, x}, v_{r, y}, \omega_{r}\right)$ between spherical ICM and Cartesian representation of the twist $\overrightarrow{t^{*}}$ are defined.

\section{Overall Control Structure}

The resulting control structure is composed by the platform control module responsible for wheel coordination and lower level wheel control modules responsible for control and coordination of steer and drive motors of each wheel module (Fig.6). The platform module converts the incoming velocity and rotation commands into the spherical representation of the ICM. Then the single components of the ICM $\rho_{\mathrm{ICM}}, \varphi_{\mathrm{ICM}}$ and $\theta_{\mathrm{ICM}}$ are controlled independently via a simple PID-controller. Based on the above derived formalisms set point values for the wheel velocity $\vec{v}_{w}$ and the wheel steering rate $\dot{\vec{\varphi}}$ are calculated and transmitted to the wheel control modules together with a prediction of the next target ICM. The wheel control modules compose the final set point values for wheel velocity $v_{w, i}$ and steering rate $\dot{\varphi}_{w, i}$ by incorporating the commanded velocity and steering rate, the control deviation between commanded ICM - converted into wheel configuration via $\vec{f}_{v_{w, i}, \varphi_{s, i}}$ - and current wheel configuration and the factor $v_{w, i, c o m p}$ compensating gear coupling and eccentric position of the drive wheel. The resulting values are then send as inputs to the motor controllers, which then independently control the steering motor and the drive motor of every wheel.

\section{RESUlts}

The above discussed control structure was implemented and simulated in a Matlab Simulink environment for the particular kinematics of Care-O-bot 3, whose mobile base is assembled from 4 independent wheel modules (Fig. 7) with off-centered orientable wheels. The platform (without additional covers) has an almost square shape with a length of approximately $60 \mathrm{~cm}$ and a width of about $50 \mathrm{~cm}$. With 

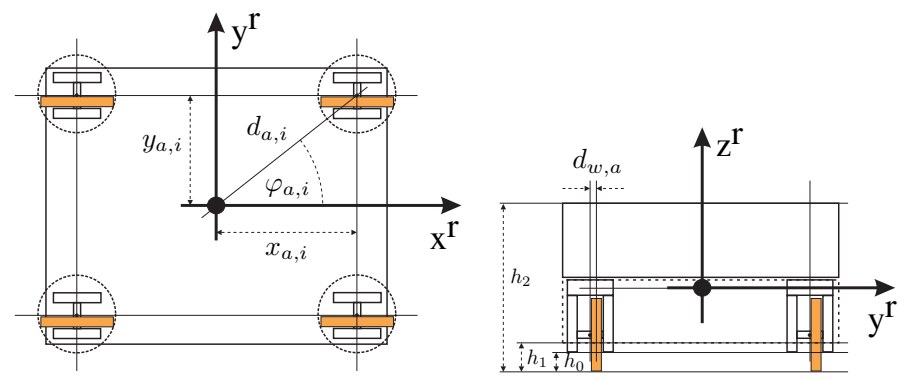

Fig. 7. Top and front view of Care-O-bot 3's mobile base

respect to the robot coordinate frame the steering-axes of the wheels lie at $x_{w, i}= \pm 23,5 \mathrm{~cm}$ and $y_{w, i}= \pm 18,5 \mathrm{~cm}$. The wheels are off-centered to the steering-axes by $d_{w, a}=$ $2,2 \mathrm{~cm}$. The chassis clearance $h_{1}$ is restricted by the accumulator - mounted underneath the robot - and is about $5 \mathrm{~cm}$ (right-hand in Fig. 7). The total height of the system $h_{2}$ is approximately $35 \mathrm{~cm}$.

Section V-A presents results obtained when implementing the different ICM representations.In section V-B the resulting steering directions $\vec{\varphi}_{s}$ and wheel steering rates $\frac{\partial}{\partial t} \vec{\varphi}_{s}$ obtained from the above described control structure are examined and compared to results obtained when the components of the twist $\vec{t}$ where directly controlled in their Cartesian representation. The simulations consider the kinematical properties of the system but do not consider any dynamical aspects.

\section{A. Characteristics in ICM Behavior}

In Fig. 8 the desired velocity components (Fig. 8(a)) for a robot driving slalom are depicted together with the resulting ICM coordinates relative to the robot in Cartesian $\left(x_{\text {IсM }}, y_{\text {IсM }}\right)$ (Fig 8(b)), polar $\left(r_{\text {IсM }}, \phi_{\text {IсM }}\right)$ (Fig 8(c)) and spherical $\left(\rho_{\text {IсM }}, \varphi_{\text {Iсм }}, \theta_{\text {IсM }}\right)$ (Fig $\left.8(d)\right)$ representation. Figure 9 shows the resulting robot path (solid blue line) in the world coordinate frame together with the corresponding trajectories of the planar ICM (red dashed line). The singularities in the common Cartesian and polar representation arising whenever the rotational rate crosses zero are clearly visible in form of the strong growth of $r_{\mathrm{ICM}}$ (Fig 8(b)) and $y_{\text {IсM }}$ (Fig 8(c)). In Fig. 9 they appear in form of the red dashed line which exit the plot on one side and reenter on the other side. In contrast to that the spherical representation stays bounded and continuously differentiable. Thus the design of an appropriate controller becomes significantly easier. Considering the opposite case, when the translational velocity takes a sinusoidal form and the rotational rate is non-zero - a rosettelike motion -, the $\theta_{\mathrm{ICM}}$ and $\phi_{\mathrm{ICM}}$ components of the spherical and polar representation show discontinuousities, while the Cartesian parameters show a continuous behavior. However, all components stay bounded and adaption of a controller to such a characteristic is far easier then to cope with infinity regions.

\section{B. Effect of ICM on wheel module set point values}

Figure 10(a) shows an arbitrary velocity command together with the resulting curves if the command is
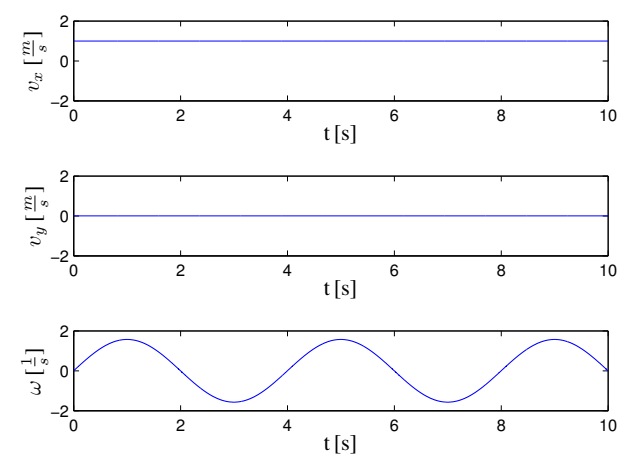

(a) Components of commanded velocity and rotation
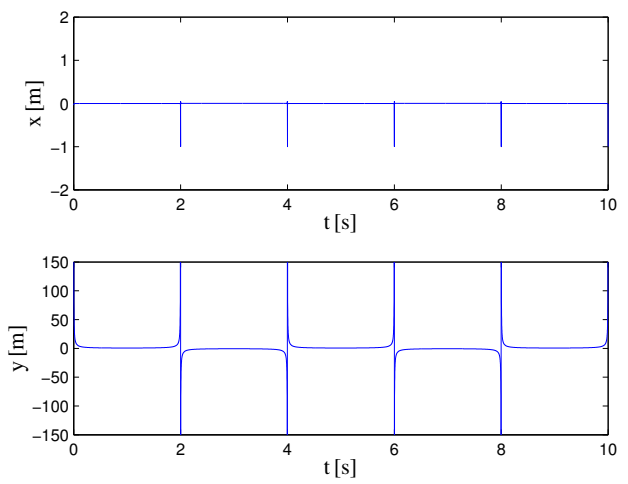

(b) Resulting ICM in Cartesian representation
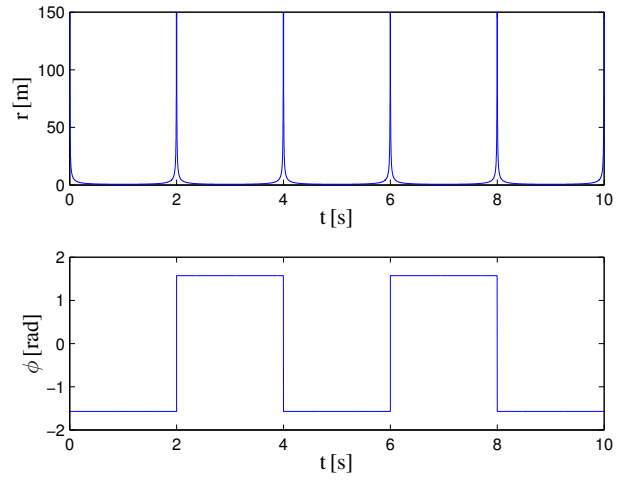

(c) Resulting ICM in polar representation
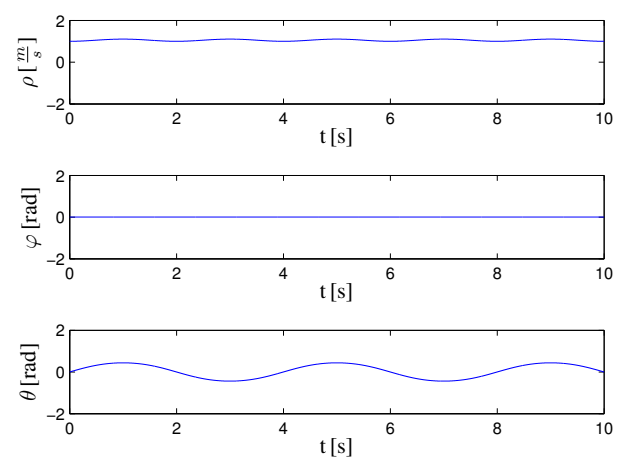

(d) Resulting ICM in spherical representation

Fig. 8. Representation induced singularities in ICM 


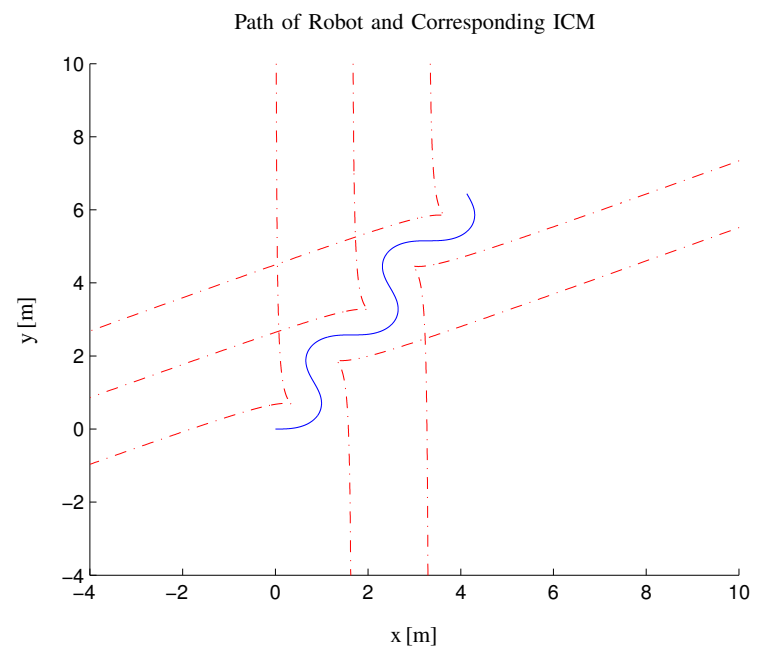

Fig. 9. Slalom path of robot (blue, solid) and corresponding ICM trajectory (red, dashed)

lowpass-filtered either directly in its Cartesian representation (Fig. 10(b)), or after transformation in its spherical representation (Fig. 10(c)) and the resulting ideal trajectories (Fig. 10(d)). The depicted command provokes critical behavior whenever either the translational velocity or the rotational velocity is close to zero while the other component changes. The corresponding set point values for the steering direction (plots 1 and 2 in Fig. 11(a)) show an discontinuous behavior and the set point values for the corresponding steering rate (plots 1 and 2 in Fig. 11(b)) grow rapidly. In fact, for the unfiltered input the steering rates would directly take infinit values. This is not observed simply because of the limited time resolution of the simulation. This behavior is due to the fact that for the platform considered the steering directions of the wheels result from the ratio between the different velocity components and not from their absolute value. Thus controlling the absolute value (Fig. 10(b)) does not remedy that problem. The steering rates are only reduced due to the lowpass, which causes the components to never reach zero after having been set to a non-zero value. In contrast to that, controlling the spherical ICM means to control the ratios of the velocity components instead of their absolute values. Thus the corresponding curve of the steering direction (plot 3 in Fig. 11(a)) changes continuously and the according steering rate (plot 3 in Fig. 11(b)) is smaller by a factor of 10 than that of the controlled Cartesian commands and smaller by a factor of 40 than that of the uncontrolled commands. The described behavior means that the platform is not able to change its state of motion instantaneously. This coincides with the platforms degree of mobility $\delta_{m}=1$ as derived in section I. The influence of the filters on the motion is depicted in Figure 10(d). The ideal path of the robot for the unfiltered Cartesian command is unsteady and cannot be realized by a pseudo-omnidirectional, non-holonomic platform without stopping and reorienting its wheels. The path corresponding to the valid filtered spherical ICM components shows a continuous, steady behavior. Figure 11(a) shows the
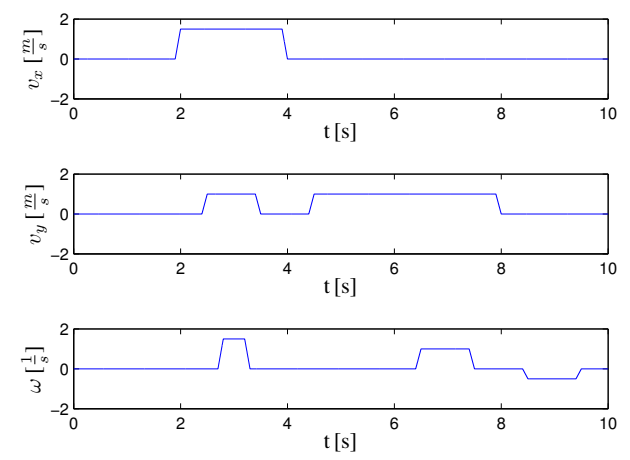

(a) Desired values of velocity and rotation
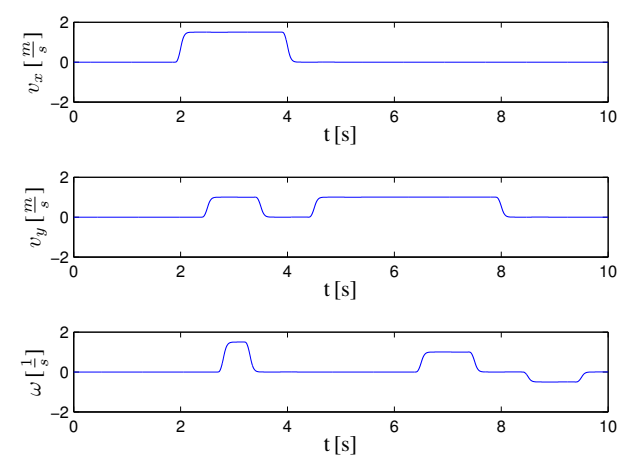

(b) Desired velocities and rotation after lowpass-filtering
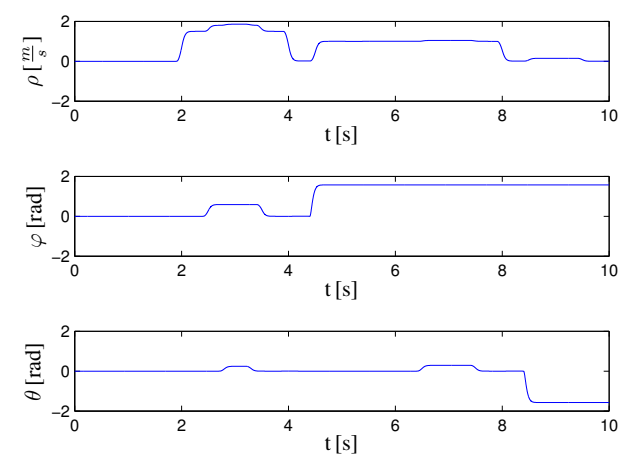

(c) ICM after transformation and lowpass-filtering

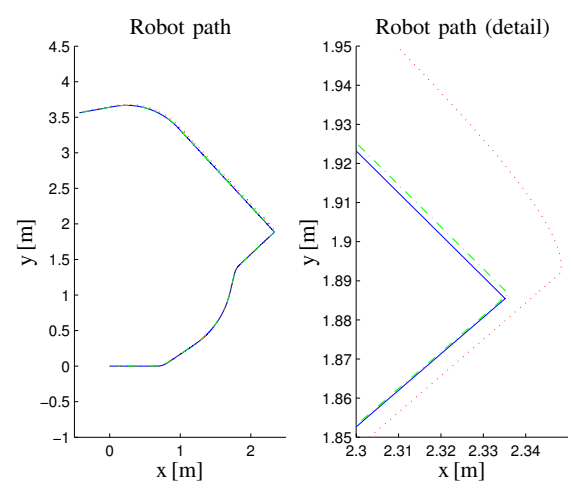

(d) Paths for unfiltered (solid), filtered cart. (dashed) and filtered spherical (dotted) commands

Fig. 10. Velocity commands and corresponding robot path 

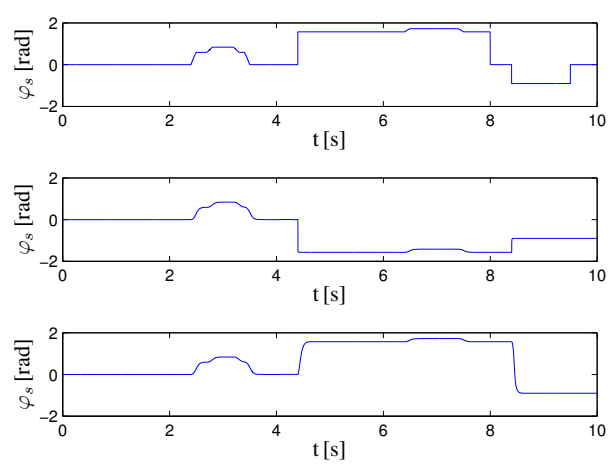

(a) Commanded direction of wheels
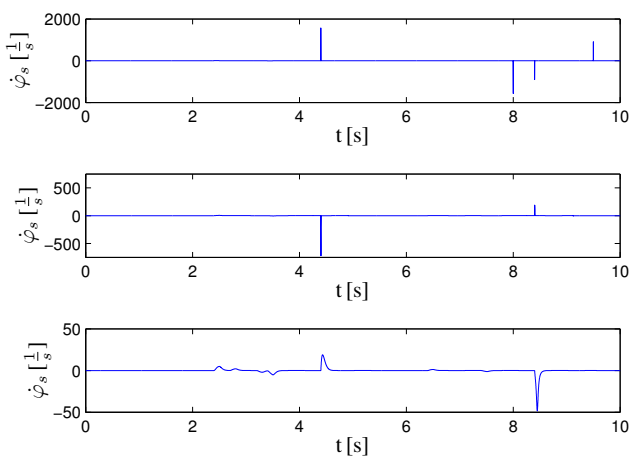

(b) Commanded change in direction of wheels

Fig. 11. Resulting set point values for steering direction and steering rate for desired twist $\vec{t}$, filtered twist and filtered spherical ICM (twist $\vec{t}^{*}$ )

branching of the solutions addressed in section IV-B. This branching appears in form of the $\pi$-difference between the desired steering direction for the time interval from 4.5 to 8.5 seconds.

\section{CONCLusion AND Future Work}

In this work the problem of wheel coordination for overactuated mobile robots is addressed. After a kinematical classification of the observed system the concept of the Instantaneous Centre of Motion (ICM) was recapitulated. Special attention was paid to the ICM's potential regarding wheel coordination, its numerical drawbacks and the corresponding implications on applied control strategies.

To overcome these numerical drawbacks and enable the application of basic control strategies the usage of an ICM representation based on spherical coordinates was motivated. It was shown that control of the ICM within this representation is literally equivalent to control of the generalized velocities in their spherical representation. This means that for the system considered the spherical coordinates form a basis of a subspace in the kinematical constraints' nullspace. Finally, a formalism that directly transforms the spherical ICM trajectory back into a trajectory within the configuration space of the mobile robot was derived.

The derived transformations were adopted to the particular mechanics of the Care-O-bot 3 system. The addressed platform control module - responsible for wheel coordination was realized in a Matlab Simulink environment. Comparison of the resulting curves for wheel steering direction and wheel steering rate proved the validity of the proposed approach and revealed its advantages over control within the Cartesian Space.

While, the proposed method delivers an efficient approach to wheel coordination by decomposing the kinematical constraints it does not yet account for other constraints, e.g. limits of the steer or drive velocities. Thus, future work will focus on investigating control strategies that allow tackling this problem.

\section{REFERENCES}

[1] B. Graf, M. Hans, R.D. Schraft, "Care-O-bot II - Development of a Next Generation Robotic Home Assistant", Autonomous Robots, Vol. 16, Issue 2, March 2004, pp.193-205

[2] J. Chestnutt, M. Lau, G. Cheung, J. Kuffner, J. Hodgins, T. Kanade, "Footstep Planning for the Honda ASIMO Humanoid", IEEE Conference on Robotics and Automation, Barcelona, April 2005, pp. 629-634

[3] Jun-Ho Oh, D. Hanson, Won-Sup Kim, Il Young Han, Ill-Woo Park, "Design of Android type Humanoid Robot Albert HUBO", IEEE International Conference on Intelligent Robots and Systems, Beijing, Oct. 2006, pp. 1428-1433

[4] R. Siegwart and I.R. Nourbakhsh, "Introduction to Autonomous Mobile Robots", Intelligent Robotics and Autonomous Agents series, MIT Press, Cambridge Massachusetts, 2004

[5] G. Campion, G.Bastin and B. D'Andréa-Novel, "Structural Properties and Classification of Kinematic and Dynamic Models of Wheeled Mobile Robots", IEEE Transactions on Robotics and Automation, Vol. 12, No. 1, Feb. 1996, pp 47-62

[6] J.-P. Laumond, "Robot Motion Planning and Control", Lecture Notes in Control and Information Sciences, Vol. 229, Springer, London, 1998

[7] P. Muir, C. Neuman, "Kinematic Modelling for Feedback control of an Omnidirectional Wheeled Mobile Robot", IEEE International Conference on Robotics and Automation, 1987, pp. 1772-1778

[8] K. Tadakuma, R. Tadakuma, "Mechanical Design of "Omni-Ball": Spherical wheel for Holonomic Motion", IEEE Conference on Automation Science and Engineering, Scottsdale, Sept. 2007, pp.788-794

[9] T. Burke and H.F. Durrant-Whyte, "Kinematics for Modular Wheeled Mobile Robots", IEEE/RSJ International Conference on Intelligent Robots and Systems, Yokohama, Jul. 1993, pp 1279-1286

[10] Y. Mori, E. Nakano, T. Takahashi and K. Takayama, "A Study on the Mechanism and Control of Omni-Directional Vehicle", IEEE/RSJ International Conference on Intelligent Robots and Systems, Vol. 1, Osaka, 1996, pp 52-59

[11] K.L. Moore and N.S. Flann, "A Six-Wheeled Omnidirectional Autonomous Mobile Robot", IEEE Control Systems Magazine, Vol. 20, Issue 6, Dec. 2000, pp 53-66

[12] D.B. Reister and M.A. Unseren, "Position and Constraint Force Control of a Vehicle with Two or More steerable Drive Wheels", IEEE Transactions on Robotics and Automation, Vol. 9, No. 6, Dec. 1993, pp 723-731

[13] M. Hashimoto, N. Suizu, I. Fujiwara and F. Oba, "Path tracking Control of a Non-Holonomic Modular Omnidirectional Vehicle", IEEE International Conference on Systems, Man and Cybernetics, Vol. 6, Tokyo, Oct. 1999, pp 637-642

[14] M. Lauria, I. Nadeau, P. Lepage, Y. Morin, P. Giguére, F. Gagnon, D. Létourneau and F. Michaud, "Design and Control of a Four Steered Wheeled Mobile Robot", IEEE 32nd Annual Conference on Industrial Electronics, Nov. 2006, pp 4020-4025

[15] B. Thuilot, B. D'Andréa-Novel and A. Micaelli, "Modeling and Feedback Control of Mobile Robots Equipped with Several Steering Wheels", IEEE Transactions on Robotics and Automation, Vol. 12, No. 3, June. 1996, pp 375-390

[16] M. Brandstötter and M. Hofbauer, "Kinematikbasierte Regelung zur Rekonfiguration fehlerbehafteter mobiler Roboter", TU Graz Diploma Thesis, Graz, Nov. 2007 